\title{
Changes in the proteomic profiles of mouse brain after infection with cyst-forming Toxoplasma gondii
}

\author{
Dong-Hui Zhou1*, Fu-Rong Zhao', Si-Yang Huang ${ }^{1}$, Min-Jun Xu', Hui-Qun Song ${ }^{1}$, Chunlei Su ${ }^{2}$ \\ and Xing-Quan Zhu ${ }^{1,3^{*}}$
}

\begin{abstract}
Background: Toxoplasma gondii is an opportunistic pathogenic protozoan parasite, which infects approximately one third of the human population worldwide, causing opportunistic zoonotic toxoplasmosis. The predilection of T. gondii for the central nervous system (CNS) causes behavioral disorders and fatal necrotizing encephalitis and thus constitutes a major threat especially to AIDS patients.

Methods: In the present study, we explored the proteomic profiles of brain tissues of the specific pathogen-free (SPF) Kunming mice at $7 \mathrm{~d}, 14 \mathrm{~d}$ and $21 \mathrm{~d}$ after infection with cysts of the Toxoplasma gondii Prugniaud (PRU) strain (Genotype II), by two-dimensional gel electrophoresis (2-DE) combined with MALDI-TOF/TOF tandem mass spectrometry (MS/MS).

Results: A total of 60 differentially expressed protein spots were selected. Fifty-six spots were successfully identified, which corresponded to 45 proteins of the mouse. Functional analysis using a Gene Ontology database showed that these proteins were mainly involved in metabolism, cell structure, signal transduction and immune responses, and will be beneficial for the understanding of molecular mechanisms of $T$. gondii pathogenesis.

Conclusions: This study identified some mouse brain proteins involved in the response with cyst-forming T. gondii PRU strain. These results provided an insight into the responsive relationship between $T$. gondii and the host brain tissues, which will shed light on our understanding of the mechanisms of pathogenesis in toxoplasmic encephalitis, and facilitate the discovery of new methods of diagnosis, prevention, control and treatment of toxoplasmic encephalopathy.
\end{abstract}

Keywords: Toxoplasma gondii, Cyst, Brain, Proteome, Two-dimensional gel electrophoresis (2-DE), Mass spectrometry (MS)

\section{Background}

The obligate intracellular parasite Toxoplasma gondii is an important water- and food-borne protozoan and can infect humans as well as almost all warm-blooded animals including mammals and birds, and the infection usually persists throughout the life of the hosts [1-5]. T. gondii infects approximately $30 \%$ of the human population worldwide

\footnotetext{
*Correspondence: donghui822002@163.com; xingquanzhu1@hotmail.com

'State Key Laboratory of Veterinary Etiological Biology, Key Laboratory of Veterinary Parasitology of Gansu Province, Lanzhou Veterinary Research Institute, Chinese Academy of Agricultural Sciences, Lanzhou, Gansu Province 730046, PR China

Full list of author information is available at the end of the article
}

and $8 \%$ of population in China [6]. Humans can be infected by ingesting tissues cysts in uncooked meat or by ingesting food and water contaminated with oocysts from infected cat feces $[1,2,6]$. There are three infectious stages of T. gondii: tachyzoites (the rapidly multiplying form), bradyzoites (latent form found in tissue cysts) and sporozoites (in oocysts) [7]. Bradyzoites develop in cysts within host cells in a number of tissues, and they are more common in neural and muscular tissues.

T. gondii has long been known as an important opportunistic pathogen of immuno-compromised patients. Toxoplasmosis ranks high on the list of diseases that

\section{Biomed Central}


lead to death of the AIDS patients. Encephalitis caused by $T$. gondii is the most predominant manifestation of toxoplasmosis in immunosuppressed patients and is now recognized with great frequency in patients treated with immunosuppressive agents [8-11].

In the past decade, proteomic approaches have been extensively employed to study the interaction between pathogens and their hosts. The most frequently used technique in quantitative proteomics is two-dimensional electrophoresis (2-DE). In quantitative 2-DE, the appropriate experimental design plays an important role in the detection of significant and reliable protein expression differences [12]. Despite that there are some limitations of the technology, such as offering a limited dynamic range of separated proteins, it has been used to investigate host cell proteome changes after infection with $T$. gondii tachyzoites [13,14], but little is currently known of the proteomic changes at differential time points in host brain tissues after infection with $T$. gondii cysts. In the present study, we applied 2-DE combined with mass spectrometry to study proteomic changes in mouse brain tissues infected with $T$. gondii cysts. The objective was to examine the proteomic modulation of host brain by cyst-forming $T$. gondii in vivo.

\section{Methods}

\section{Ethics statement}

The present study was approved by the Animal Ethics Committee of Lanzhou Veterinary Research Institute, Chinese Academy of Agricultural Sciences (Approval No. LVRIAEC2010-008). The mice were handled in accordance with good animal practices required by the Animal Ethics Procedures and Guidelines of the People's Republic of China.

\section{Sample collection}

Sixty Specific-Pathogen-Free (SPF)-grade female Kunming mice (including 30 mice for T. gondii infection and 30 mice as non-infected control), aged 6 to 8 weeks old, were purchased from Sun Yat-Sen University Laboratory Animal Center. All mice were handled in accordance with good animal practice according to the Animal Ethics Procedures and Guidelines of the People's Republic of China. All mice were maintained under standard conventional conditions, with food and water ad libitum.

T. gondii (PRU) strain (Genotype II) was kindly provided by Prof Hai-Zhu Zhang (Department of Parasitology, Xinxiang Medical College, Henan, China) and were preserved in our laboratory. Tissue cysts of the PRU strain were obtained from the brains of Kunming mice infected with cysts according to Yan et al. [15]. Cysts were counted under an optical microscope. After counting, each of the 30 mice in the treatment group was inoculated intra-gastrically with 10 cysts of the PRU strain, while 30 mice in the non-infection control group were inoculated intra-gastrically with sterile physiological saline. After inoculation, the mice were observed daily for clinical symptoms. Based on a previous study [16] and our pilot study, at $7 \mathrm{~d}, 14 \mathrm{~d}$ and $21 \mathrm{~d}$ post infection, six mice from both treatment group and the control group were euthanized and the whole brain of each mouse was rapidly removed separately, washed to remove the blood, and immediately stored at $-80^{\circ} \mathrm{C}$ until proteomic analysis, or directly processed for protein extraction. The mice that were successfully infected and produced T. gondii cysts were determined by observing tissue cysts in the brain under an optical microscope (for $14 \mathrm{~d}$ and $21 \mathrm{~d}$ ).

\section{Protein extraction}

Proteins were prepared according to the previously published protocol [13]. In brief, mouse brain tissues of six mice from each group, respectively, were lysed in lysis buffer containing $7 \mathrm{M}$ urea, 2\% CHAPS \{3-[(3cholamidopropyl)-dimethylammonio]-1-propanesulfonate\}, $2 \mathrm{M}$ thiourea (Amersham), $20 \mathrm{mM}$ Tris- $\mathrm{HCl}$ ( $\mathrm{pH} 8.5$, Amresco) and phenylmethylsulfonyl fluoride solution (Amresco). Then the sample was sonicated on ice $(80 \mathrm{~W}$, $12 \mathrm{~s}$ duration, 10 times, with $2 \mathrm{~min}$ intervals) and centrifuged at $12,000 \times g$ for $20 \mathrm{~min}$ at $4^{\circ} \mathrm{C}$. The supernatant was transferred to a new centrifuge tube with four times the volume of acetone added. The mixture was then precipitated overnight at $-20^{\circ} \mathrm{C}$ and centrifuged on the next day with the same parameters. The precipitate was harvested and stored at $-80^{\circ} \mathrm{C}$ until use. The samples were prepared in triplicates.

\section{Isoelectric focusing electrophoresis}

2-DE procedure was performed essentially according to a protocol published previously [13]. Briefly, before the isoelectric focusing electrophoresis, the precipitate was dissolved in rehydration buffer containing $7 \mathrm{M}$ urea, 2\% CHAPS, $2 \mathrm{M}$ thiourea, followed by centrifugation at $12,000 \times g$ for $20 \mathrm{~min}$ at $4^{\circ} \mathrm{C}$. The protein concentration was determined by the Bradford method using a $2 \mathrm{D}$ Quant kit (Amresco) according to the manufacturer's instructions. Using an equal mixture of the brain tissue protein of each mouse in the control group and treatment group respectively, proteins were initially separated using an Ettan IPGphor 3 Isoelectric Focusing system (GE Healthcare). Brain tissue proteins were focused to their isoelectric points on a $24 \mathrm{~cm}(\mathrm{pH} \mathrm{4-7)} \mathrm{Immobiline}$ DryStrip (GE Healthcare) with the following parameters: $300 \mathrm{~V}$ for $20 \mathrm{~min}, 700 \mathrm{~V}$ for $30 \mathrm{~min}, 1,500 \mathrm{~V}$ for $1.5 \mathrm{~h}$, $9,000 \mathrm{~V}$ for $3 \mathrm{~h}$ and 9,000 $\mathrm{V}$ for $4 \mathrm{~h}$. After the isoelectric focusing, the IPG strip was equilibrated for $15 \mathrm{~min}$ in equilibration buffer containing $2 \%$ sodium dodecyl sulfate (SDS), $50 \mathrm{mM}$ Tris- $\mathrm{HCl}$ (pH 8.8), $6 \mathrm{M}$ urea, 30\% 
(vol/vol) glycerol, 0.002\% bromophenol blue and $100 \mathrm{mM}$ dithiothreitol (freshly added before use, Amresco), and followed by a second wash for 15 min with equilibration buffer containing $250 \mathrm{mM}$ iodoacetamide (freshly added before use, Amersham). The IPG strip was then embedded in a precast gel and sealed into place using agarose sealing solution.

\section{SDS-PAGE}

After equilibration, the immobilized $\mathrm{pH}$ gradient strips were loaded onto $12.5 \%(\mathrm{w} / \mathrm{v})$ homogeneous acrylamide gels $(1 \mathrm{~mm} \times 24 \mathrm{~cm} \times 19 \mathrm{~cm})$ and sealed with $1 \%(\mathrm{w} / \mathrm{v})$ agarose. Proteins were separated by running the gels at $2 \mathrm{~W} /$ gel for $45 \mathrm{~min}$ and then at $18 \mathrm{~W} /$ gel at $10^{\circ} \mathrm{C}$ until the bromochlorophenol blue reached the end of gels. Finally, the gels were fixed in fixing solution (ethanol: glacial acetic acid: deionized water $=4: 1: 5)$ for $2 \mathrm{~h}$, stained with Coomassie Brilliant Blue G-250 overnight and rinsed with deionized water.

\section{Analysis of gels}

Images of gels were obtained at $150 \mathrm{dpi}$ (dots/in) using a scanner (Powerlook1100, UMAX) and analyzed using ImageMaster $^{\mathrm{TM}}$ 2D Platinum 5.0 software (GE Healthcare). Spots were detected by 2DElite Automatic Spot Detection Program, which calculated spot volumes relative to the background and normalization. The volume percentage of each spot was determined by comparison of the spot volume to the total volume presented in the 2-DE gel. To select differentially expressed protein spots, quantitative analysis was performed using the Student's $t$-test by the volume percentage of spots between brain tissues of the infected and uninfected groups on the triplicate gels. Two spots were considered significantly different if $P<0.05$ and with 1.5 fold differences in volume. Spots meeting these criteria were then selected and subjected to in-gel tryptic digestion.

\section{Protein enzymolysis}

The differentially expressed protein spots were manually excised from the Coomassie Brilliant Blue-stained gels and put into a 96-well microplate. The gel pieces were washed twice with MilliQ water, destained with 50\% methanol at $37^{\circ} \mathrm{C}$ for $30 \mathrm{~min}$, and dehydrated in $100 \mu \mathrm{l}$ of acetonitrile $(\mathrm{ACN})$ at room temperature for $20 \mathrm{~min}$. Next, the samples were swollen in $50 \mu \mathrm{l}$ of $100 \mathrm{mM}$ $\mathrm{NH}_{4} \mathrm{HCO}_{3}$, dehydrated for the second time and incubated in $1 \mu \mathrm{g} / 50 \mu \mathrm{l}$ trypsin (Promega) at $4^{\circ} \mathrm{C}$ for $30 \mathrm{~min}$. Then the samples were added with coverage solution (10\% $\mathrm{ACN}, 50 \mathrm{mM} \mathrm{NH}_{4} \mathrm{HCO}_{3}$, MilliQ water) and incubated at $37^{\circ} \mathrm{C}$ for $16 \mathrm{~h}$. After suction of the coverage solution, the peptide mixtures were extracted using $2.5 \%$ trifluoroacetic (TFA)/90\% ACN at room temperature for $30 \mathrm{~min}$ and vacuum dried.

\section{Identification of protein spots by MS}

After vacuum drying, material was dissolved in $1.5 \mu \mathrm{l}$ solution containing MilliQ water, $50 \%$ ACN and $0.1 \%$ TFA. Then, $0.8 \mu \mathrm{l}$ of the mixture was loaded onto a target plate with $0.5 \mu \mathrm{l} \mathrm{HCCA}(5 \mathrm{mg} / \mathrm{ml}$ a-Cyano-4-hydroxycinnamic acid) matrix, dried at room temperature and analyzed using ABI 4800 matrix-assisted laser desorption ionization-time of flight/time of flight (MALDI-TOF/ TOF) Proteomics Analyzer mass spectrometer (Applied Biosystems, USA). The UV laser was operated at a $200 \mathrm{~Hz}$ repetition rate with a wavelength of $355 \mathrm{~nm}$ and an accelerated voltage of $20 \mathrm{kV}$.

\section{Database searching}

The experimental MS data were matched to a corresponding virtual peptide mass database derived from GPS Explorer ${ }^{\mathrm{TM}} \mathrm{v}$ 3.6, the Mascot and the International Protein Index (IPI) mouse protein database. Protein identification was carried out by peptide mass fingerprint (PMF) using the Mascot software (http://www. matrixscience.com). The search parameters used in PMF were as follows, database: IPI mouse (56871 sequences); species: mouse; enzyme: trypsin; fixed modifications: carbamidomethylation; variable modifications: oxidation (M). The function, gene name, and Gene Ontology category of each protein were determined using the Mascot v 2.1 software protein database search engine and the IPI mouse protein database.

\section{Quantitative real-time PCR verification}

Total RNA was extracted from the mouse brain tissues infected with $T$. gondii cysts using the Trizol reagent (Invitrogen). One microgram of total RNA was used to synthesize the first-strand CDNA, which was diluted 20 fold; $5 \mu \mathrm{l}$ of the diluted cDNA was used as a template for real time PCR. SYBR Green-monitored real time PCR was performed on an ABI PRISM ${ }^{\bullet} 7500$ Sequence Detection System (Applied Biosystems). The primer sequences used for real time PCR are listed in Table 1. Glyceraldehyde-3-phosphate dehydrogenase (GAPDH) was used as a housekeeping gene for the tests.

\section{Results}

\section{Comparative proteomic analysis by 2-DE}

Global protein components of mouse brain tissues were separated with 2-DE in a $24 \mathrm{~cm}, \mathrm{pH} 4-7$ IPG strip. The $2-\mathrm{DE}$ gels were processed by silver staining or Coomassie brilliant blue G-250 staining, then scanned using a UMAX scanner. Consistency of the method was confirmed by analyzing in gels in triplicate. The data were analyzed using ImageMaster ${ }^{\mathrm{TM}} 2 \mathrm{D}$ Platinum 5.0 software. The results showed that at least 2500 protein spots were detected in each gel. Spots with significant increase (or decrease) in their relative abundance were considered 


\begin{tabular}{|c|c|c|}
\hline Target genes & Primers $\left(5^{\prime}\right.$ to $\left.3^{\prime}\right)$ & $\begin{array}{l}\text { Amplicon } \\
\text { length (bp) }\end{array}$ \\
\hline \multirow[t]{2}{*}{ Calreticulin } & FP: CACCAAGAAGGTTCATGTCA & \multirow[t]{2}{*}{190} \\
\hline & RP: CAGAAAGTCCCAATCATCCT & \\
\hline \multirow{2}{*}{$\begin{array}{l}\text { Rho GDP-dissociation } \\
\text { inhibitor } 1\end{array}$} & FP: AGTCTTGTGACCCCGGAAGT & \multirow[t]{2}{*}{129} \\
\hline & RP: TCTGCCATGCTTACCTCTAGC & \\
\hline \multirow[t]{2}{*}{ Endoplasmin } & FP: ACCAGACACCAAGGCGTATG & \multirow[t]{2}{*}{150} \\
\hline & RP: TCTCCCTCATCCTGCTCTGA & \\
\hline \multirow[t]{2}{*}{ Stomatin-like protein 2} & FP: GTCAGCGCATTCTCCAAACT & \multirow[t]{2}{*}{180} \\
\hline & RP: CGTGTCTGTAGCCTGGACAT & \\
\hline \multirow[t]{2}{*}{ GAPDH } & FP: CGGCCTCCAAGGAGTAAGAAA & \multirow[t]{2}{*}{141} \\
\hline & RP: GCCCCTCCTGTTATTATGG & \\
\hline
\end{tabular}

FP: Forward primer; RP: Reverse primer.

the differentially expressed proteins if $P<0.05$ and two spots have 1.5 fold differences in volume. After bioinformatics analysis, 60 differentially expressed proteins were selected and identified using MALDI-TOF MS.

\section{Identification of differentially expressed proteins}

Proteins with MW ranging from 17 to $170 \mathrm{kDa}$ and $\mathrm{pI}$ between 4 and 7 were separated well. Differentially expressed proteins were picked from gels and identified using MALDI-TOF MS. The data are summarized in Table 2. Sixty significantly and consistently up- or downregulated protein spots with 1.5 fold changes of volume intensity in the triplicate gels were digested by trypsin and analyzed by MALDI-TOF MS.

Fifty-six of 60 differentially expressed proteins were successfully identified (Figure 1), corresponding to 45 unique proteins. These proteins are listed in Table 2. Several different protein spots were identified as the same proteins by MS. Spots 4 and 18 were identified as the same protein. Spots 22 and 44 were identified as the same protein. Spots 31 and 36 were identified as different isoforms of the same protein. At $7 \mathrm{~d}$ post $T$. gondii infection, 9 spots (spots 1-9) were down-regulated and 6 were up-regulated (spots 10-15) in mouse brain tissue. At $14 \mathrm{~d}$ post infection, 17 spots were down-regulated (spots 16-24, 39-46) and 13 were up-regulated (spots $25-27,47-56)$. At $21 \mathrm{~d}$ post infection, 13 protein spots (spots 28-32, 39-46) were down-regulated and 16 (spots 33-38, 47-56) were up-regulated. Eight protein spots (spots 39-46) and 10 protein spots (spots 47-56) were down- or up-regulated on both $14 \mathrm{~d}$ and $21 \mathrm{~d}$, respectively (Figure 1).

\section{Analysis of differentially expressed proteins}

According to the Uniprot Knowledgebase (Swiss-Prot/ TrEMBL) and Gene Ontology database, the functions of successfully identified proteins were grouped based on biological processes, cellular component and molecular function. The results showed that the function of these proteins involved in cellular metabolism, structural molecule activity, immune responses, biological regulation, metabolic process, binding, catalytic activity, enzyme regulator activity, transporter activity and other functions. The differentially expressed proteins were mainly located in the cytoplasm, cell membranes and organelles (mitochondria and lysosomes), and some are secreted proteins (Figure 2).

\section{Quantitative real-time PCR verification of differentially expressed proteins}

Four genes corresponding to the protein spots designated calreticulin, Rho GDP-dissociation inhibitor 1, endoplasmin and stomatin-like protein 2 were chosen for quantitative real-time PCR analysis to quantify their transcript levels. The real time PCR results were consistent with those of the 2-DE studies, and suggested that these proteins identified as differentially expressed were regulated at transcriptional level.

\section{Discussion}

The present study compared the proteomic profiles of brain tissues at $7 \mathrm{~d}, 14 \mathrm{~d}$ and $21 \mathrm{~d}$ after infection with cysts of $T$. gondii PRU strain by 2-DE analysis. A total of 60 differentially expressed protein spots were selected and identified by MALDI-TOF MS. Of these, 56 protein spots were successfully identified, which represented 45 different proteins. Four protein spots were not successfully identified, which may be due to the low concentrations of the proteins, which therefore failed to produce high quality mass spectrometric data.

GO analysis revealed that most of the differentially expressed proteins are involved in metabolism, cell structure, signal transduction and immune responses. Here, we focused on the discussion of the functions of several main differentially expressed proteins, and the relationships between these proteins and $T$. gondii infection.

Serine protease inhibitor (SERPIN) A3k is persistently up-regulated in mouse brain tissues 7,14 and 21 days after infection with Toxoplasma cysts. Many members of the serine protease inhibitor superfamily play an important role in the physiological and pathological process, and can be regarded as protease inhibitors which are involved in the coagulation reaction, fiber dissolution, angiogenesis, complement activation, immune and inflammatory reaction $[17,18]$. It was suggested that SERPINs could inhibit host cell apoptosis [19]. In addition, SERPIN may inhibit replication and decrease T. gondii viability [20]. Therefore, the persistence of upregulated SERPIN A3k may play an important role in preventing the death of infected mouse brain cells as 
Table 2 Differentially expressed proteins between $T$. gondii-infected and non-infected mouse brain tissues

Spot Accession Protein name Score Expect Queries Sequence Nominal Calculated Matched peptides

matched Coverage mass

Fold change Functional

No. No.

pl value

(Infected/non- categories

infected)

Day 7, down-regulated proteins

$1 \quad$ IPI00230394 Lamin-B1

$288 \quad 9.00 \mathrm{E}-25 \quad 27 \quad 36$

$66973 \quad 5.11$

ASAPATPLSPTR:LQEKEELR:SLETENSALQ

$-2.91$

Structural molecule

LQVTEREEVR;ALYETELADARR;LREYEAAL

NSK;VDLENRCQSLTEDLEFRKNMYEEEINE

TRR;LVEVDSGR;LAQALHEMREQHDAQVR

LYKEELEQTYHAK;LALDMEISAYR;LKNTSE

QDQPMGGWEMIR;NQNSWGTGEDVK;NS

GGEEVAQR;TTIPEEEEEEEEEPIGVAVEEERF

HOQGAPR

218

25

$45201 \quad 5.99$

SRPGCCAGPRGYR:NVFLSPMSISSALAMVF MGAK:TGTOYLLR:FYEAELEFLDFOGATEES R;FIEWTR;LGMTDAFGGR;AFVEVNEEGTEA AAATAGMMTVR

3 IPI00110721 Isoform 1 of Glyoxalase domain-containing

$508 \quad 9.00 \mathrm{E}-47 \quad 12$

33581

protein 4

$4 \quad$ IPI00113660 Proteasome activator complex subunit 3

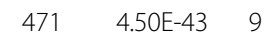

42

$29602 \quad 5.69$

FQTVHFFRDVLGMQVLRHEEFEEGCK;LGN

DFMGITLASSQAVSNAR;VAEGIFETEAPGG

YKFYLQDR;IYEQDEEKQR;LELQGIQGAVD

HAAAFGR:ELPDLEDLMKR:LLDDAMEADK

SDEWFATR

ITSEAEDLVANFFPK:RLDECEEAFQGTK;SN

QOLVDIIEKVKPEIR:IEDGNNFGVSIOEETVA

ELRTVESEAASYLDQISRYYITR;YPHVEDYR

RTVTEIDEKEYISLR

5 IPI00130344 Chloride intracellular channel protein 1

$3.60 \mathrm{E}-16 \quad 6$

39

$27338 \quad 5.09$

GNCPFSOR;YPKLAALNPESNTSGLDIFAK VLDNYLTSPLPEEVDETSAEDEGISOR:GFTI PEAFR:YLSNAYAREEFASTCPDDEEIELAYE QVAR

$6 \quad$ IPI00121851 Prefoldin subunit 3

$7 \quad$ IPI00315794 Cytochrome b5

$212 \quad 3.60 \mathrm{E}-17$

16365

FLLADNLYCK:NLDSLEEDLDFLR:VYNWD VKR

VEGSEPSVTYYR:NSAEETWMVIHGRVYDITR FLSEHPGGEEVLLEQAGADATESFEDVGHS PDAR:OYYIGDVHPSDLKPK

$8 \quad$ P|00124501 Galectin-related protein $\mathrm{A}$

9 IPI00308984 Eukaryotic translation initiation factor $1 \mathrm{~A}$

Day 7, up-regulated proteins

$10 \quad$ IPI00131830 Serine protease inhibitor A3K

LDDGHLNNSLGSPVQADVYFPRLIVPFCGH IK;AVFTDR;NSCISGERGEEQSAIPYFPFIPDQ PFRVEILCEHPR:VFVDGHOLFDFYHRIQTLS AIDTIK

ELVFKEDGQEYAQVIK;LEAMCFDGVKR:WW INTSDIILVGLR

Cell part and

organelle

Enzyme regulator activity

Transporter activity

Metabolic process

Binding and

enzyme activator

activity

Binding

DLQILAEFHEK,ALYQTEAFTADFQQPTEAK;

ELISELDER;ISFDPQDTFESEFYLDEKR;HFRD EELSCSVLELK;MQQVEASLQPETLRK;FSIAS NYR;AVLDVAETGTEAAAATGVIGGIR 
Table 2 Differentially expressed proteins between $T$. gondii-infected and non-infected mouse brain tissues (Continued)

\begin{tabular}{|c|c|c|c|c|c|c|c|c|c|c|c|}
\hline 11 & IPI00131830 & $\begin{array}{l}\text { Serine protease } \\
\text { inhibitor A3K }\end{array}$ & 511 & $4.50 \mathrm{E}-47$ & 12 & 35 & 47021 & 5.05 & $\begin{array}{l}\text { DLQILAEFHEK;ALYQTEAFTADFQQPTEAK; } \\
\text { ELISELDER;ISFDPQDTFESEFYLDEKR;HFRD } \\
\text { EELSCSVLELK;MQQVEASLQPETLRK;FSIAS } \\
\text { NYRLEEDVLPEMGIKEVFTEQADLSGITETK; } \\
\text { AVLDVAETGTEAAAATGVIGGIR }\end{array}$ & 3.15 & $\begin{array}{l}\text { Enzyme regulator } \\
\text { activity }\end{array}$ \\
\hline 12 & IPI00131830 & $\begin{array}{l}\text { Serine protease } \\
\text { inhibitor A3K }\end{array}$ & 463 & $2.90 \mathrm{E}-42$ & 10 & 28 & 47021 & 5.05 & $\begin{array}{l}\text { DLQILAEFHEK;ALYQTEAFTADFQQPTEAK; } \\
\text { ELISELDER;ISFDPQDTFESEFYLDEKR;HFRD } \\
\text { EELSCSVLELK;MQQVEASLQPETLRK;FSIAS } \\
\text { NYR;AVLDVAETGTEAAAATGVIGGIR }\end{array}$ & 6.30 & $\begin{array}{l}\text { Enzyme regulator } \\
\text { activity }\end{array}$ \\
\hline 13 & IPI00131830 & $\begin{array}{l}\text { Serine protease } \\
\text { inhibitor A3K }\end{array}$ & 564 & $2.30 \mathrm{E}-52$ & 14 & 35 & 47021 & 5.05 & $\begin{array}{l}\text { DLQILAEFHEK;ALYQTEAFTADFQQPTEAK; } \\
\text { ELISELDER;ISFDPQDTFESEFYLDEKR;HFRD } \\
\text { EELSCSVLELK;MQQVEASLQPETLRK;FSIAS } \\
\text { NYRLEEDVLPEMGIKEVFTEQADLSGITETKK; } \\
\text { AVLDVAETGTEAAAATGVIGGIR }\end{array}$ & 4.02 & $\begin{array}{l}\text { Enzyme regulator } \\
\text { activity }\end{array}$ \\
\hline 14 & IPI00131830 & $\begin{array}{l}\text { Serine protease } \\
\text { inhibitor A3K }\end{array}$ & 596 & $1.40 \mathrm{E}-55$ & 12 & 35 & 47021 & 5.05 & $\begin{array}{l}\text { DLQILAEFHEK;ALYQTEAFTADFQQPTEAK; } \\
\text { ELISELDER;/ISFDPQDTFESEFYLDEKR;HFRD } \\
\text { EELSCSVLELK;MQQVEASLQPETLRK;FSIAS } \\
\text { NYRLEEDVLPEMGIKEVFTEQADLSGITETK; } \\
\text { AVLDVAETGTEAAATGVIGGIR }\end{array}$ & 5.80 & $\begin{array}{l}\text { Enzyme regulator } \\
\text { activity }\end{array}$ \\
\hline 15 & IPI00310972 & $\begin{array}{l}\text { GTP-GDP dissociation } \\
\text { stimulator } 1 \text { isoform b }\end{array}$ & 490 & $5.70 \mathrm{E}-45$ & 18 & 27 & 61381 & 5.28 & $\begin{array}{l}\text { NEFMR;DQEVLLQTGR;EQFASTNIAEELVK; } \\
\text { QIEHDKREMIFEVLAPAENDAIK;LMDLLD } \\
\text { RHVEDGNVTVQHAALSALR;SEMPPVQFK; } \\
\text { MLIDAQAEAAEQLGK;LVEWCEAKDHAGV } \\
\text { MGESNR;DLASAQLVQILHRLLADER;SVAQ } \\
\text { QASLTEQR }\end{array}$ & 8.32 & $\begin{array}{l}\text { Cell part and } \\
\text { organelle part }\end{array}$ \\
\hline \multicolumn{12}{|c|}{ Day 14 , down-regulated proteins } \\
\hline 16 & IPI00131871 & $\begin{array}{l}\text { COP9 signalosome } \\
\text { complex subunit } 4\end{array}$ & 449 & $7.20 \mathrm{E}-41$ & 18 & 50 & 46541 & 5.57 & $\begin{array}{l}\text { QLLTDFCTHLPNLPDSTAK;VISFEEQVASIR } \\
\text { QHLASIYEKEEDWRNAAQVLVGIPLETGQK } \\
\text { QYNVDYKLETYLK;LLEDDDPVQAEAYINR } \\
\text { ASLLQNESTNEQLQIHYK;KFIEAAQR;TIVHE } \\
\text { SERLEALKHALHCTILASAGQQR;MLATLFK } \\
\text { DERCQQLAAYGILEK;ATTADGSILDR;IAS } \\
\text { QMITEGRMNGFIDQIDGIVHFETR }\end{array}$ & -2.50 & Metabolic process \\
\hline 17 & IPI00338039 & Tubulin beta-2A chain & 591 & 4.50E-55 & 22 & 42 & 50274 & 4.78 & $\begin{array}{l}\text { MREIVHIQAGQCGNQIGAKFWEVISDEHGI } \\
\text { DPTGSYHGDSDLQLERINVYYNEAAGNK; } \\
\text { AILVDLEPGTMDSVR;GHYTEGAELVDSVLD } \\
\text { WRK;IREEYPDRIMNTFSVMPSPK;FPGQLN } \\
\text { ADLRKLAVNMVPFPRLHFFMPGFAPLTSRG } \\
\text { SQQYRALTVPELTQQMFDSKNMMAACD } \\
\text { PR;ISEQFTAMFRR }\end{array}$ & -1.90 & $\begin{array}{l}\text { Structural molecule } \\
\text { activity }\end{array}$ \\
\hline 18 & IPI00113660 & $\begin{array}{l}\text { Proteasome activator } \\
\text { complex subunit } 3\end{array}$ & 450 & $5.70 \mathrm{E}-41$ & 11 & 43 & 29602 & 5.69 & $\begin{array}{l}\text { ERITSEAEDLVANFFPK:RLDECEEAFQGTK;S } \\
\text { NQQLVDIIEKVKPEIR;EDGNNFGVSIQEETV } \\
\text { AELRTVESEAASYLDQISRYYITR;YPHVEDYR } \\
\text { RTVTEIDEKEYISLR }\end{array}$ & -2.04 & $\begin{array}{l}\text { Enzyme regulator } \\
\text { activity }\end{array}$ \\
\hline 19 & IPI00407019 & $\begin{array}{l}\text { Tetratricopeptide } \\
\text { repeat protein } 19\end{array}$ & 348 & $9.00 E-31$ & 12 & 23 & 41550 & 5.87 & $\begin{array}{l}\text { LSIMKDEPEAAELILHDALRLAYESDNRK;GQ } \\
\text { LENAEQLFK;QLSQAQR;ALQICQEIQGER;EI } \\
\text { YQEALKR;RDEVSVQHIREELAELSR }\end{array}$ & -1.99 & $\begin{array}{l}\text { Cell part, organelle } \\
\text { and organelle part }\end{array}$ \\
\hline
\end{tabular}


Table 2 Differentially expressed proteins between $\boldsymbol{T}$. gondii-infected and non-infected mouse brain tissues (Continued)

\section{8}

21

IPI00313962 Ubiquitin carboxylterminal hydrolase

isozyme L1

22

IPI00322312 Rho GDP-dissociation

inhibitor 1

$23 \quad$ IPI00845712 Isoform 1 of UPF0424 protein C1orf128 homolog

24

$$
\text { IPI00221826 Isoform Short of }
$$

Splicing factor,

arginine/serine-rich 3

\section{Day 14, up-regulated proteins}

$25 \quad$ IPI00123613 Protein kinase C and casein kinase substrate in neurons protein 1 sirtuin-2

$27 \quad$ IPI00649438 Novel protein similar to splicing factor, arginine/serine-rich 3

\section{Day 21, down-regulated proteins}

$28 \quad$ IPI00133522 Protein disulfideisomerase
$1.40 \mathrm{E}-49 \quad 12$

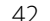

29859

5.57

AVIFDRFR:DLONVVITLRILFRPVASOLPRIY

K;FDAGELITQR;Q

GDGLIELRKLEAAEDIAYQLSR

LGVAGQWR;QIEELKGQEVSPK;QFLSETEKL SPEDR:CFEKNEAIQAAHDSVAQEGQCR:M PFPVNHGASSEDSLIODAKKEFTEREOGE VR

SIOEIOELDKDDESLR;YKEALLGRVAVSADP NVPNVIVTR:EGVEYR;YIQHTYR:IDKTDYMV GSYGPRAEEYEFLTPMEEAPK;FTDDDKTD GSYGPRAEEYEF

CAAEREEPPEQRGLAYGLYLR;LQCLNESR: GVFKPWEER;FVESDADEELLFNIPFTGNVK LKGVIIMGEDDDSHPSEMR;NIPQMSFDDT EREPEOTFSLNRDITGELEYATK;IFYIGLRGE EREPEQTFSLNRDITGELEYATK;IFYIGLRGE
WTELRRHEVTICNYEASANPADHRVHQVTP QTHFIS;

DSCPLDCK:AFGYYGPLRSWWARNPPGFA FVEFEDPR;NRGPPPSWGR;DDYRR

$-4.73$

Metabolic process

Cell proliferation

Enzyme regulator activity

Cell part, organelle and organelle part

$\begin{array}{ll}-2.40 & \text { Cell part, organelle } \\ \text { and organelle part }\end{array}$

LCNDLMSCVQER;AYAQQLTDWAKR;AWG AMMTEADKVSELHQEVK;AYHLACKEER:TT POYMEGMEQVFEOCOOFEEKR:HLNLAEN PQYMEGMEQVFEQCQQFEEKR;HLNLAEN
SSYMHVYRELEQAIRGADAOEDLR:KAEGA SSYMHVYRELEQAIRGADAQEDLR;KAEGA TLSNATGAVESTSQAGDR;ALYDYDGQEQ
DELSFK;LGEEDEQGWCRGRLDSGQLGLYP ANYVEAI

NLFTQTLGLGSQK;LLDELTLEGVTRYMQSER; 1.57 VICLVGAGISTSAGIPDFR;HPEPFFALAK;CY TQNIDTLER;IFSEATPRCEQCQSWKPDIVFF GENLPSR;APLATPR;ELEDLVRREHANIDAQ SGSQAPNPSTTISPGK

NPPGFAFVEFEDPRDAADAVR;NRGLPPSW 1.65

Catalytic activity and binding

Catalytic activity and binding

Metabolic process

SNFEEALAAHK-ALAPEYAKR:LKAEGSEIR:V DATEESDLAQQYGVR;YQLDKDGWLFKKF DEGRNNFEGEITK;HNQLPLVIEFTEQTAPK; ILFIFIDSDHTDNQR;KEECPAVR;YKPESDELT AEKITEFCHR;|KPHLMSQEVPEDWDKQPVK VLVGANFEEVAFDEKK;LGETYKDHENIIIAK; FFPASADR 
Table 2 Differentially expressed proteins between $\boldsymbol{T}$. gondii-infected and non-infected mouse brain tissues (Continued)

endophilin B2

IPI00111556 Seri

threonine-protein 239

phosphatase 2A catalytic

subunit beta isoform

31 IPI00798544 Isoform 2 of Enolasephosphatase E1

$32 \quad$ PI00230145 Ferritin heavy chain

Day 21, up-regulated proteins

34 IPI00221540 Erlin-2

35 IPI00230192 Isoform Alpha-1 of Guanine nucleotidebinding protein $\mathrm{G}(\mathrm{O})$ subunit alpha

36 PI00653664 Isoform 1 of Enolasephosphatase E1

$37 \quad$ IPI00130280 ATP synthase subunit alpha
KLASDAGIFFTR;FGQAEKTELDAHFENLLAR; $\quad-1.50$

NWTER.OTEVLLOPNPSAR.ATCEGDTVPDF

QETRPRVAQTEFDROAEVTRLLLEGSSTHV

NHLR:SOTTYYAQCYR

SPDTNYLFMGDYVDR;QITQVYGFYDECLR

KYGNANWWK:LQEVPHEGPMCDLLWSDP

DDRGGWGISPR:AHOLVMEGYNWCHDRN

WTIFSAPNYCYR;YSFLQFDPAPR

QLQGHMWK:MKAEFFADWPAVRRWREA $\quad-3.54$

GMKYYIYSSGSVEAQK.GHKVDSE

QNYHQDAEAAINR;YFLHQSHEEREHAEK; $\quad-1.56 \quad$ Immune system

KPDRDDWESGLNAMECALHLEKSVNOSL

ELHKLATDKNDPHLCDFIETYYLSEQ

LELHATDKNDPHLCDFIETYYS

GHGDES

AVFVDLEPTVIDEVR;EIIDLVLDR;NLDIERPT YTNLNR;FDGALNVDLTEFOTNLVPYPRIHF PLATYAPVISAEK

SVQTTLQTDEVK;VTKPNIPEAIRR;VAQVAEI TYGQK;KISEIEDAAFLAR;AKADAECYTALK

AMDTLGVEYGDKER;LWGDSGIQECFNR;YY 1.54 LDSLDRIGAGDYQPTEQDILR;LFDVGGQR

Structural molecule activity

Metabolic process

Signal transducer activity

EYLQTHWEEEECQQDVSLLR;QLQGHMW K:MKAEFFADWPAVRRWREAGMKVYIYSS GSVEAQK;VDSESYRK

TGTAEMSSILEERILGADTSVDLEETGRVLSI GDGIAR;NVQAEEMVEFSSGLK;TGAIVDVPV GEELLGR;APGIIPRISVREPMQTGIKAVDSLV PIGR;ELI IGDR

AQHLHQLAADTYKEFERAYIPEGQR;EEAQ QRTDMELLR;IFTNSLMFGTSDR;LKDLEEG QALMOELEDGSPR:OTYDKFDANMR:AE YLR;RFVESSCAF 
Table 2 Differentially expressed proteins between $T$. gondii-infected and non-infected mouse brain tissues (Continued)

Spots 39-46 were down-regulated on both day 14 and day 21

GTSGIDAKKTSCEFTGDILRTPVSEDMLGR;

GPWLAEDFLDIMGQPINPQCRIYPEEM

IQTGISAIDGMNSIAR:IPIFSAAGLPHNEIAA QICR:SKDWDYSEENFAIVFAAMGVNMET AR;LALTTAEFLAYQCEKHVLVILTDMSSYA EALR;RGFPGYMYTDLATIYER;QIYPPINV LPSLSRLMKSAIGEGMTR;DHADVSNQLYA CYAIGK;AWGEEALTSDDLLYLEFLQK;NFIT QGPYENRTVYETLDIGWQLLR;RIPQSTLSEF YPR

GFAFVQYVNER;MYSYPARVPPPPPIAR

C1/C2

$41 \quad$ IPI00115117 Stomatin-like protein 2

NTVILFVPQQEAWVER;ILEPGLNVLIPVLD R;ASYGVEDPEYAVTQLAQTTMR;YEIKDIHV PPRVKESMQMQVEAER;ATVLESEGTR;APV PGAQNSSQSRRDVQATDTSIEELGR protein Cops6

IPI00311515 Beta-soluble NSF attachment protein

SQEGRPMQVIGALIGK;NIEVMNSFELLSHT VEEK;QVCEIIESPLFLK;IGVDHVAR;LILEYVKA SEAGEVPFNHEILR:TCNTMNQFVNKFNVLY

EAVQLMAEAEKR;ASHSFLRGLFGGNTRIEE ACEMYTR;NWSAAGNAFCQAAK;HDSATS FVDAGNAYK:HHITIAEIYETELVDIEKAIAHY EOSADYYKGEESNSSANK:VAAYAAOLEOY OKAIEIYEOVGANTMDNPLLK:AALCHFIVD ELNAKLALEKYEEMFPAFTDSRLLEAHEEQN SEAYTEAVKEFDSISRLDQWLTTMLLR; inhibitor 1

$45 \quad$ IPI00133853 UPF0687 protein C20orf27 homolog

46 IPI00315504 ADP-ribosylation factor-like protein 2

SIQEIQELDKDDESLR;YKEALLGRVAVSADP NVPNVIVTR;EGVEYR;YIQHTYR;IDKTDYM GSYGPR;FTDDDKTDHLSWEWNLTIK

FAAGHDAEGSQSHVHFDEK;YEITFTLPPVR. ETPVHSLHLKLLSVTPTSEGYSIK;EGVLKEEM LLACEGDIGTCVR;HHGTPMLLDGVK

Cell part and organelle part

ELQSLLVEER;SHHWR 
Table 2 Differentially expressed proteins between $T$. gondii-infected and non-infected mouse brain tissues (Continued)

\section{Spots 47-56 were up-regulated on both day 14 and day 21}

$47 \quad$ IPI00129526 Endoplasmin

TDDEWQREEEAIQLDGLNASQIR;FAFQAE VNR;NKEIFLRELISNASDALDKIRLISLTDENA LAGNEELTVK:NLLHVTDTGVGMTREELVKE VEEDEYKAFYK;ESDDPMAYIHFTAEGEVTF KSILFVPTSAPRGLFDEYGSKK:RVFITDDFHD MMPKYLNFVKGWDSDDLPLNVSR;IADEK YNDTFWK;LGVIEDHSNR;FQSSHHSTDITSL YNDTFWK;LGVIEDHSNR;FQSSHHSTDITSL
DOYVER:ODKIYFMAGSSRKEAESSPFVERE DQYVER;QDKIYFMAGSSRKEAESSPFVERE
ATEKEFEPLLNWMK;LTESPCALVASQYGW SGNMER;DISTNYYASQKKTFEINPR;AYGDR A3K A3K A3K

$1.40 \mathrm{E}-27 \quad 8$

26 A3K

$1.10 \mathrm{E}-14 \quad 5$ protein Otub1

EQFLDGDAWTNR;HEQNIDCGGGYVK;IDN SQVESGSLEDDWDFLPPKKIKDPDAAKPED WDER;GEWKPR

ALYQTEAFTADFQQPTEAK;ISFDPQDTFES EFYLDEKR;HFRDEELSCSVLELK;MQQVEAS LQPETLRK;AVLDVAETGTEAAAATGVIGGIR; GKTMEEILEGLK;DLQILAEFHEK;ALYQTEAF TADFQQPTEAK;ISFDPQDTFESEFYLDEKR; HFRDEELSCSVLELK;MQQVEASLQPETLRK; AVLDVAETGTEAAAATGVIGGIR

DLQILAEFHEK;ALYQTEAFTADFQQPTEAK ;ISFDPQDTFESEFYLDEKR;HFRDEELSCSVL ELK;MQQVEASLQPETLRK;FSIASNYR;AVLD VAETGTEAAAATGVIGGIR

ISFDPQDTFESEFYLDEKR;HFRDEELSCSVL ELK:MQQVEASLQPETLR;AVLDVAETGTEA AAATGVIGGIR

APLVPDIPSADPGPGPAASRGGTAVILDIFR; KVYEGGSNVDQFVTR;YGGPTPPYIPNHK;TL SFDLQQRLSDEEGTNMHLQLVRQEMAVCP EQLSEFLDSLR;NCFHVAAVR

IQQEIAVQNPLVSER;EYAEDDNIYQQK;YSYI RKTRPDGNCFYR;LLTSGYLQR;FFEHFIEGGR

FWDYLR.ELEEOL GPVAEETR:LGADMEDLR; NEVHTMLGQSTEEIR;LSTHLR;LGPLVEQGR; TANLGAGAAQPLR;AQAFGDR;GRLEEVGN QAR;SKMEEQTQQIRLQAEIFQAR;GWFEPIV EDMHR 

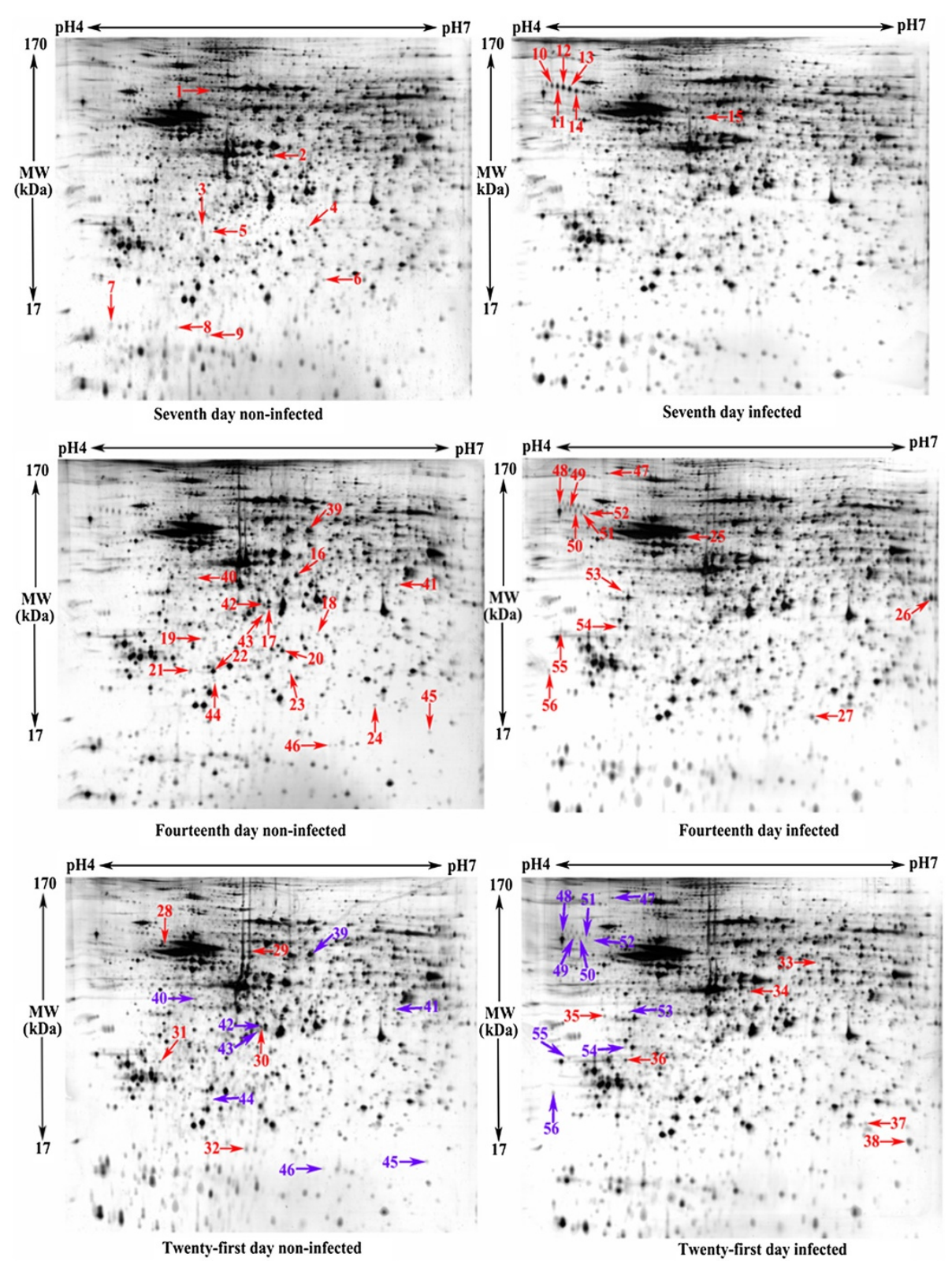

Figure 1 Two-dimensional electrophoresis profiles of infected and non-infected mouse brain tissues with or without cyst-forming Toxoplasma gondii.

well as limiting the growth of $T$. gondii in the brain tissue cysts.

Protein disulfide isomerase (PDI) is down-regulated 14 days after infection with T. gondii cysts. PDI family members can function as molecular chaperones and as disulfide oxidoreductase/isomerases, which means that they can make, break, or rearrange disulfide bonds [21]. The PDI family's main function is to catalyze the oxidative folding of nascent polypeptide chains in the endoplasmic reticulum, yet they also play an important role in the ERassociated protein degradation pathway (ERAD), protein transport, calcium homeostasis, antigen presentation and virus invasion [22,23]. PDI may play an important role in immune and inflammatory responses and one of the intracellular effector molecules involved in anti-inflammatory reactions [24].

Endoplasmin, also known as heat shock protein 90B1, is a molecular chaperone protein. In the present study, it showed sustained up-regulation in brain tissues 14 and 21 days after infection with $T$. gondii. In Toll like receptor and integrin secretory pathways, it plays a crucial role in folding protein, so was thought to be one of the basic immune chaperone proteins in the regulation of innate and adaptive immunity [25]. Calreticulin (CRT) is 


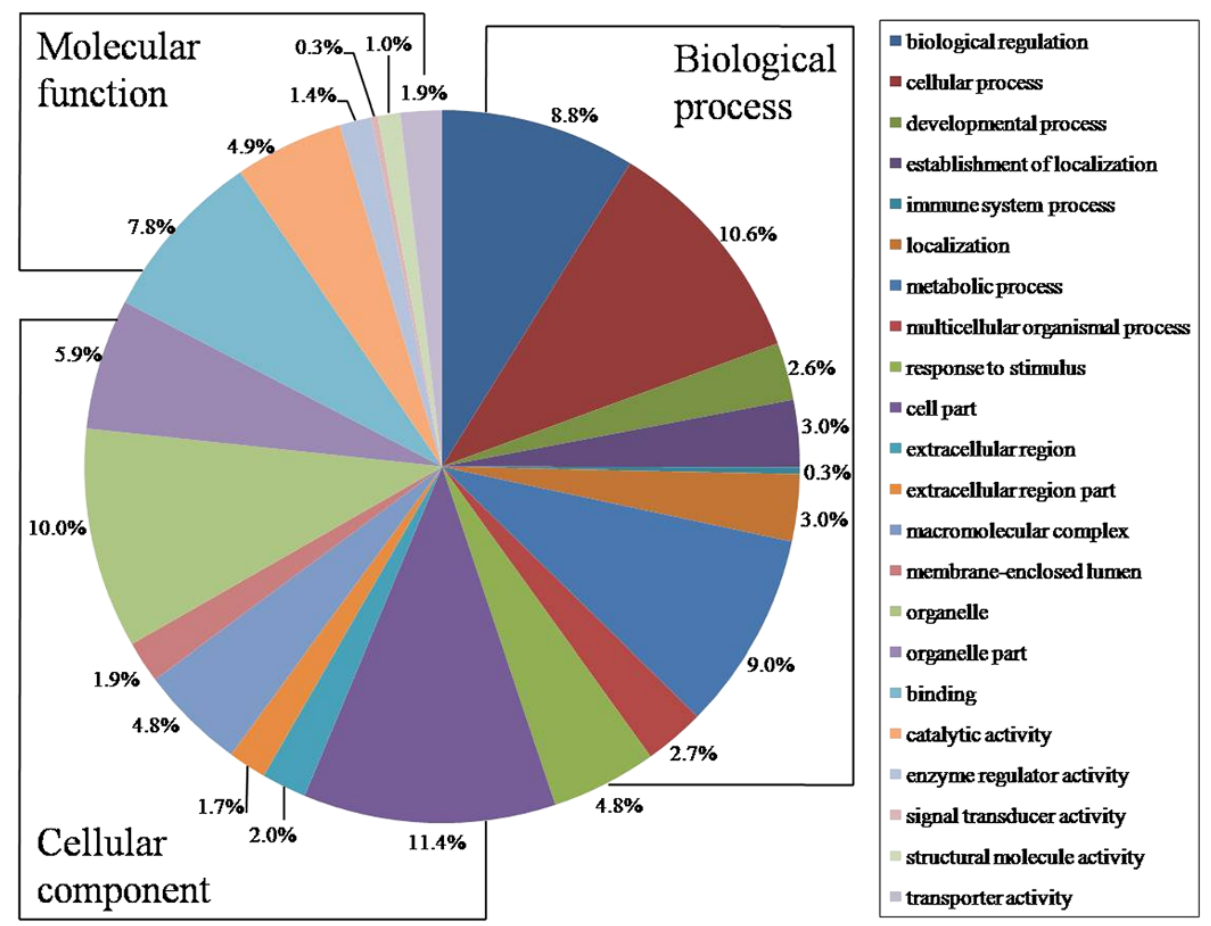

Figure 2 Gene ontology (GO) categories of the identified proteins were obtained from the Uniprot Web site (http://www.uniprot.org/), classified into cellular component, molecular function, and biological process according to the GO terms.

persistently up-regulated in brain tissues 14 and 21 days after infection with Toxoplasma cysts. Calreticulin is a $\mathrm{Ca}^{2+}$ binding protein that has been implicated in many diverse functions inside and outside of the endoplasmic reticulum (ER), including the regulation of intracellular $\mathrm{Ca}^{2+}$ homeostasis and $\mathrm{Ca}^{2+}$-dependent pathways, chaperone activity, steroid-mediated gene expression, cell adhesion and the interactions of CRT with immunoglobulin G and immunoglobulin Y [26-28]. CRT could be used as a biomarker in lung cancer prediction and diagnosis [29]. CRT also plays an important role in autoimmunity. Enolase 1 and CRT are important proteins in regulating the differentiation and functions of mouse mast cells [30]. CRT has important implications involved in the genesis, development and prognosis of many diseases.

Lamin B1 is down-regulated in mouse brain tissues 7 days after infection with $T$. gondii cysts. Lamins are the important cytoskeletal proteins in the nucleus, and can be divided into two types A and B. Lamin B1 is necessary for growth, development and nuclear membrane integrity in mice [31-33]. Cytochrome $b_{5}$ is also downregulated in mouse brain tissues 7 days after infection with $T$. gondii cysts. It is a small microsomal protein which serves as an electron transfer component in a number of oxidative reactions in biological tissues, including the anabolic metabolism of fats and steroids, as well as the catabolism of xenobiotics and compounds of endogenous metabolism [34].
Prohibitin (PHB) is down-regulated in mouse brain tissues 14 days after infection with $T$. gondii cysts. PHB has a variety of cell biological functions, including the regulation of cell proliferation, apoptosis, development, transcription, mitochondrial protein folding and as a cell surface receptor [35-37]. In the present study, $\alpha$-tubulin of mouse brain tissues is up-regulated after infection with $T$. gondii cysts at 21 days post infection, but $\beta$-tubulin is down-regulated at 14 days post infection. $\alpha$ - and $\beta$-tubulins are the major components of microtubules of the eukaryotic cytoskeleton. Microtubules constitute a major portion of cytoplasmic proteins in nerve cells. They have been implicated to play a central role in axonal transport, neurotransmitter release, neurite outgrowth and synaptogenesis. An increase in the tubulin microheterogeneity was demonstrated during brain maturation. Tubulin comprises a large percentage of the total protein in brain [38-40]. Therefore, abnormal expression of tubulin could have an effect on the normal growth and development of host brain, even causing behavioral disorders of the host.

Apolipoprotein E (ApoE) is persistently up-regulated in brain tissues at 14 and 21 days after infection with Toxoplasma cysts. ApoE is one of components of plasma lipoprotein. It regulates the metabolism of plasma lipoprotein by binding to lipoprotein receptors [41]. Abnormal expression of the protein causes the disorder of the metabolism of plasma lipoprotein, and is closely related 
to atherosclerosis, hypercholesterolemia and hypertriglyceridemia [42].

\section{Conclusions}

The present study revealed changes in the proteomic profiles of mouse brain tissues after infection with cystforming T. gondii PRU strain (Genotype II). 45 mouse brain proteins differentially expressed between infected and non-infected mice were identified, these proteins were mainly involved in metabolism, cell structure, signal transduction and immune responses. Further exploration of these proteomic data will contribute to understanding the pathogenesis of toxoplasmic encephalitis, and facilitates the discovery of new methods of diagnosis, prevention, control and treatment of Toxoplasma encephalopathy.

\section{Competing interests}

The authors declare that they have no competing interests.

\section{Authors' contributions}

$\mathrm{XQZ}$ and $\mathrm{DHZ}$ conceived and designed the study, and critically revised the manuscript. DHZ, FRZ and SYH performed the experiments, analyzed the data and drafted the manuscript. MJX, HQS and CS helped in study design, study implementation and manuscript revision. All authors read and approved the final manuscript.

\section{Acknowledgments}

Project support was provided by the National Natural Science Foundation of China (Grant Nos. 31230073, 31172316 and 31228022). We thank Guangzhou FitGene Biotechnology Co., Ltd., China, for technical assistance.

\section{Author details \\ 'State Key Laboratory of Veterinary Etiological Biology, Key Laboratory of Veterinary Parasitology of Gansu Province, Lanzhou Veterinary Research Institute, Chinese Academy of Agricultural Sciences, Lanzhou, Gansu Province 730046, PR China. 'Department of Microbiology, The University of Tennessee, Knoxville, TN 37996, USA. ${ }^{3}$ College of Animal Science and Veterinary Medicine, Heilongjiang Bayi Agricultural University, Daqing, Heilongjiang Province 163319, PR China.}

Received: 18 February 2013 Accepted: 6 April 2013

Published: 12 April 2013

\section{References}

1. Dubey JP: Toxoplasmosis of Animals and Humans. secondth edition. Boca Raton, Florida: CRC Press; 2010:313.

2. Dubey JP, Jones JL: Toxoplasma gondii infection in humans and animals in the United States. Int J Parasitol 2008, 38:1257-1278.

3. Nardoni S, Angelici MC, Mugnaini L, Mancianti F: Prevalence of Toxoplasma gondii infection in Myocastor coypus in a protected Italian wetland. Parasit Vectors 2011, 4:240.

4. Chen J, Xu MJ, Zhou DH, Song $H Q$, Wang $C R$, Zhu XQ: Canine and feline parasitic zoonoses in China. Parasit Vectors 2012, 5:152.

5. Tian YM, Dai FY, Huang SY, Deng ZH, Duan G, Zhou DH, Yang JF, Weng YB, Zhu $X Q$, Zou FC: First report of Toxoplasma gondii seroprevalence in peafowls in Yunnan Province, Southwestern China. Parasit Vectors 2012, 5:205.

6. Zhou P, Chen Z, Li HL, Zheng H, He S, Lin RQ, Zhu XQ: Toxoplasma gondii infection in humans in China. Parasit Vectors 2011, 4:165.

7. Dubey JP: History of the discovery of the life cycle of Toxoplasma gondii. Int J Parasitol 2009, 39:877-882.

8. da Silva RC, Langoni H: Toxoplasma gondii: host-parasite interaction and behavior manipulation. Parasitol Res 2009, 105:893-898.

9. Suzuki Y: Host resistance in the brain against Toxoplasma gondii. J Infect Dis 2002, 185(Suppl 1):S58-65.
10. Afonso C, Paixão VB, Costa RM: Chronic Toxoplasma infection modifies the structure and the risk of host behavior. PLOS One 2012, 7:e32489.

11. Carruthers VB, Suzuki Y: Effects of Toxoplasma gondii infection on the brain. Schizophr Bull 2007, 33:745-751.

12. Tchankouo-Nguetcheu S, Bourguet E, Lenormand P, Rousselle JC, Namane A, Choumet V: Infection by chikungunya virus modulates the expression of several proteins in Aedes aegypti salivary glands. Parasit Vectors 2012, 5:264.

13. Zhou DH, Yuan ZG, Zhao FR, Li HL, Zhou Y, Lin RQ, Zou FC, Song HQ, $\mathrm{XU}$ MJ, Zhu XQ: Modulation of mouse macrophage proteome induced by Toxoplasma gondii tachyzoites in vivo. Parasitol Res 2011, 109:1637-1646.

14. Nelson MM, Jones AR, Carmen JC, Sinai AP, Burchmore R, Wastling JM: Modulation of the host cell proteome by the intracellular apicomplexan parasite Toxoplasma gondii. Infect Immun 2008, 76:828-844.

15. Yan HK, Yuan ZG, Song HQ, Petersen E, Zhou Y, Ren D, Zhou DH, Li HX, Lin $R Q$, Yang GL, Zhu XQ: Vaccination with a DNA vaccine coding for perforin-like protein 1 and MIC6 induces significant protective immunity against Toxoplasma gondii. Clin Vaccine Immunol 2012, 19:684-689.

16. Djurković-Djaković O, Djokić V, Vujanić M, Zivković T, Bobić B, Nikolić A, Slavić K, Klun I, Ivović V: Kinetics of parasite burdens in blood and tissues during murine toxoplasmosis. Exp Parasitol 2012, 131:372-376.

17. Silverman GA, Bird PI, Carrell RW, Church FC, Coughlin PB, Gettins PG, Irving JA, Lomas DA, Luke CJ, Moyer RW, Pemberton PA, Remold-O'Donnell E, Salvesen GS, Travis J, Whisstock JC: The serpins are an expanding superfamily of structurally similar but functionally diverse proteins. Evolution, mechanism of inhibition, novel functions, and a revised nomenclature. J Biol Chem 2001, 276:33293-33296.

18. Molehin AJ, Gobert GN, McManus DP: Serine protease inhibitors of parasitic helminths. Parasitology 2012, 139:681-695.

19. Ahn HJ, Kim JY, Ryu K, Nam HW: STAT6 activation by Toxoplasma gondii infection induces the expression of Th2 C-C chemokine ligands and B clade serine protease inhibitors in macrophage. Parasitol Res 2009 105:1445-1453.

20. Shaw MK, Roos DS, Tilney LG: Cysteine and serine protease inhibitors block intracellular development and disrupt the secretory pathway of Toxoplasma gondii. Microb Infect 2002, 4:119-132.

21. Benham AM: The protein disulfide isomerase family: key players in health and disease. Antioxid Redox Signal 2012, 16:781-789.

22. Wilkinson B, Gilbert HF: Protein disulfide isomerase. Biochim Biophys Acta 2004, 1699:35-44.

23. Stolf BS, Smyrnias I, Lopes LR, Vendramin A, Goto H, Laurindo FR, Shah AM, Santos CX: Protein disulfide isomerase and host-pathogen interaction. SCi World J 2011, 11:1749-1761.

24. Higuchi T, Watanabe $Y$, Waga I: Protein disulfide isomerase suppresses the transcriptional activity of NF-kappaB. Biochem Biophys Res Commun 2004, 318:46-52.

25. Morales C, Wu S, Yang Y, Hao B, Li Z: Drosophila glycoprotein 93 is an ortholog of mammalian heat shock protein gp96 (grp94, HSP90b1, HSP4) and retains disulfide bond-independent chaperone function for TLRs and integrins. J Immunol 2009, 183:5121-5128.

26. Wang WA, Groenendyk J, Michalak M: Calreticulin signaling in health and disease. Int J Biochem Cell Biol 2012, 44:842-846.

27. Gelebart P, Opas M, Michalak M: Calreticulin, a Ca2 +-binding chaperone of the endoplasmic reticulum. Int J Biochem Cell Biol 2005, 37:260-266.

28. Møllegaard KM, Duus K, Træholt SD, Thaysen-Andersen M, Liu Y, Palma AS, Feizi T, Hansen PR, Højrup P, Houen G: The interactions of calreticulin with immunoglobulin G and immunoglobulin Y. Biochim Biophys Acta 1814, 2011:889-899

29. Liu R, Gong J, Chen J, Li Q, Song C, Zhang J, Li Y, Liu Z, Dong Y, Chen L, Jin B: Calreticulin as a potential diagnostic biomarker for lung cancer. Cancer Immunol Immunother 2012, 61:855-864.

30. Ryu SY, Hong GU, Kim DY, Ro JY: Enolase 1 and calreticulin regulate the differentiation and function of mouse mast cells. Cell Signal 2012, 24:60-70.

31. Shimi T, Butin-Israeli V, Adam SA, Hamanaka RB, Goldman AE, Lucas CA, Shumaker DK, Kosak ST, Chandel NS, Goldman RD: The role of nuclear lamin B1 in cell proliferation and senescence. Genes Dev 2011, 25:2579-2593.

32. Vergnes L, Péterfy $M$, Bergo MO, Young SG, Reue K: Lamin B1 is required for mouse development and nuclear integrity. Proc Natl Acad Sci USA 2004, 101:10428-10433.

33. Martin C, Chen S, Maya-Mendoza A, Lovric J, Sims PF, Jackson DA: Lamin B1 maintains the functional plasticity of nucleoli. J Cell Sci 2009, 122:1551-1562. 
34. Schenkman JB, Jansson I: The many roles of cytochrome b5. Pharmacol Ther 2003, 97:139-152.

35. Van Aken O, Whelan J, Van Breusegem F: Prohibitins: mitochondrial partners in development and stress response. Trends Plant Sci 2010, 15:275-282

36. Theiss AL, Sitaraman SV: The role and therapeutic potential of prohibitin in disease. Biochim Biophys Acta 1813, 2011:1137-1143.

37. Mishra S, Ande SR, Nyomba BL: The role of prohibitin in cell signaling. FEBS J 2010, 277:3937-3946.

38. George HJ, Misra L, Field DJ, Lee JC: Polymorphism of brain tubulin. Biochemistry 1981, 20:2402-2409.

39. Eipper BA: Properties of rat brain tubulin. J Biol Chem 1974, 249:1407-1416.

40. Gozes I, Littauer UZ: Tubulin microheterogeneity increases with rat brain maturation. Nature 1978, 276:411-413.

41. Hauser PS, Narayanaswami V, Ryan RO: Apolipoprotein E: from lipid transport to neurobiology. Prog Lipid Res 2011, 50:62-74.

42. Gregg RE, Zech LA, Schaefer EJ, Stark D, Wilson D, Brewer HB Jr: Abnormal in vivo metabolism of apolipoprotein E4 in humans. J Clin Invest 1986, 78:815-821.

doi:10.1186/1756-3305-6-96

Cite this article as: Zhou et al:: Changes in the proteomic profiles of mouse brain after infection with cyst-forming Toxoplasma gondii. Parasites \& Vectors 2013 6:96.

\section{Submit your next manuscript to BioMed Central and take full advantage of:}

- Convenient online submission

- Thorough peer review

- No space constraints or color figure charges

- Immediate publication on acceptance

- Inclusion in PubMed, CAS, Scopus and Google Scholar

- Research which is freely available for redistribution 